\title{
Where old players meet new regulators
}

White, PJ. et al. Cell Metab 27, 1281-1293 (2018)

In today's world of cheap, sugary foods, endomorphy has become common with two in three American adults classified as overweight or obese. Glucose and lipid dysregulation are notorious biomarkers of these and other related disorders. However, less well known to the general public, but highly predictive, are changes in circulating branched-chained amino acids (BCAAs), which includes the proteinogenic amino acids leucine, isoleucine, and valine. Evidence of elevated BCAAs in obese humans was first reported half a century ago, and while other studies since then have validated the obesity/BCAA correlation and expanded the collective knowledge of this linkage, many details remain unclear. However, a new paper from Prof. Christopher Newgard's group at the Duke Molecular Physiology Institute, published in the journal Cell Metabolism, sheds light on the biochemistry of these processes and their regulation.

Based on rodent and human studies, it appears a significant portion of this increase in circulating BCAAs is attributable to decreased breakdown of BCAAs in liver tissue. This reduction in catabolism is due to a decline in activity of the protein assembly responsible for BCAA catabolism, known as branched-chain ketoacid dehydrogenase complex (BCKDH). This enzymatic slowdown results from increases in a specific phosphorylation event on one of the complex proteins, acting like a brake on BCAA catabolism. In turn, the BCKDH phosphosite is known to be regulated by two enzymes: the BCKDH kinase (BDK) adds the phosphate moiety, while Protein Phosphatase, Mg2+/Mn2+ Dependent $1 \mathrm{~K}$ (PPM1K) turns up enzyme action by removing the phosphate.

Because the metabolism of BCAAs in liver tissue is reduced, investigators decided to see what would happen if they reopened the floodgates, by revving up BCAA catabolism. For the study, they selected fatty Zucker rats, which have a missense mutation in the leptin receptor and will eat till corpulent. As such, they are often used in studies of diet-induced obesity, insulin resistance, and metabolic syndrome. Additionally, notes first author Phillip White, Zuckers accumulate BCAAs in blood without dietary supplementation much like overweight humans, something missing from other animal models of obesity.

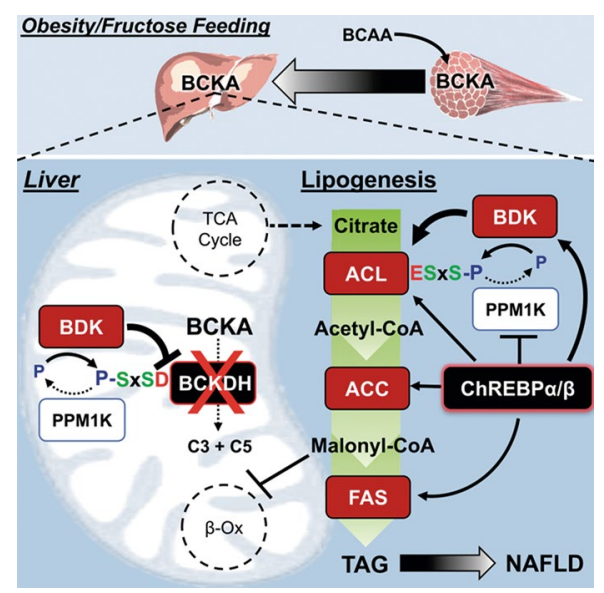

BCAA metabolism in liver is regulated by the kinase, BDK, and phosphatase, PPM1K, at key phosphosites. Reprinted with permission from White et al. (2018), Elsevier.

The authors used two independent approaches to increase BCAA breakdown in liver. First, working with collaborators David Chuang and Max Wynn at the University of Texas Southwestern Medical Center, who had developed a small molecule inhibitor of BDK, they pharmacologically retarded $\mathrm{BDK}$ action with the intention to decrease phosphorylation of $\mathrm{BCKDH}$, thereby increasing enzyme function. Investigators also used virally mediated liverspecific overexpression of human PPM1K in the fatty Zuckers to alter phosphorylation levels at the key phosphosite.

Over a seven day time course, rats receiving the inhibitor had increased BCKDH activity across different tissues, resulting in decreased BCAA and associated metabolite levels. Investigators also observed decreases in hepatic triglycerides, suggesting increased catabolism of fatty acids, and improved responses to glucose tolerance testing. Upregulation of the phosphatase produced the same promising results.

Given the significant and diverse effects these treatments had on metabolism in fatty rats, the Duke team speculated that there might be other targets for BDK and PPM1K. To determine this, they conducted phosphoproteomic screens of liver extracts to identify other phosphosites that were altered in liver tissue of animals subjected to either approach. Several phosphosites changed, but besides the original site on $\mathrm{BCKDH}$, only one other changed with both treatments, meaning co-regulation by both $\mathrm{BDK}$ and PPM1K. The new phosphosite was a serine residue on ATP-citrate lyase (ACL), a central enzyme involved in lipogenesis. For ACL, phosphorylation on this particular site increases enzymatic activity.

Based on phosphosites identified on other proteins, additional experiments, and informatic analysis, the investigators hypothesized that BDK and PPM1K were part of a lipogenic circuit regulated by the transcription factor carbohydrate response element binding protein (ChREBP). To test this hypothesis, the Newgard lab collaborated with Mark Herman's group at Duke, experts in ChREBP metabolism. Together they overexpressed the ChREBP- $\beta$ isoform in liver of healthy lean rats; as predicted, BDK levels increased while PPM1K decreased, establishing a link between an important transcriptional regulator of lipogenesis and elevated BCAAs in obesity. Based on this relationship and the glucose activation of ChREBP, it follows that people on a Western diet would experience elevated lipogenesis, associated dyslipidemia, and elevated BCAAs.

White, who was a postdoc in Newgard's group and now runs his own lab at Duke, believes this work opens up a new metabolic regulatory node, saying "We can go in and kind of normalize three of the macronutrients that are altered in obesity and insulin resistant states by going after one specific kinase/phosphate axis," He adds, "A strength of the study is that it really begins with retro-translation, which is a theme of our institute, where we aim to start with unbiased observations in human populations of disease and then try to work backwards to understand what those observations mean.”

He's cautiously optimistic about the possibility for translation of these findings into a new therapeutic approach because these studies were based on human observations and White and colleagues already have a potent small molecule inhibitor in hand. He acknowledged the need for additional studies to check for longterm toxicity issues and efficacy for the drug within other models and in longer-term treatment paradigms.

\section{Clark Nelson}

Published online: 25 June 2018 https://doi.org/10.1038/s41684-018-0102-z 